\title{
The effect of physiotherapeutic intervention on the static balance of elderly for secondary prevention of falls
}

\begin{abstract}
lakovidis T. Paris ${ }^{1 *}$, Markopoulos Nikolaos ${ }^{2}$, Kottaras Stavros ${ }^{3}$, Mavromoustakos Savvas ${ }^{4}$, Apostolou Thomas ${ }^{5}$, Kallistratos Ilias $^{6}$ and Takidis loannis ${ }^{7}$
\end{abstract}

*Correspondence: paris_physio@yahoo.com

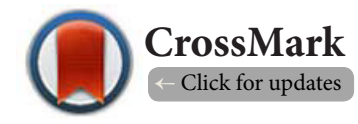

'Paris lakovidis Lecturer A.T.E.I. Thessaloniki, Greece.

${ }^{2}$ Apostolou Thomas MD ,Orthopedic Surgeon, Assosiate Professor ATEl of Thessaloniki, Greece.

${ }^{3}$ Markopoulos Nikolaos, Orthopedic surgeon G.H.Kilkis, Greece.

${ }^{4}$ Mavromoustakos Savvas, Assistant professor A.T.E.I. Thessaloniki, Greece.

${ }^{5}$ Kottaras Stavros, Assistant professor A.T.E.I. Thessaloniki, Greece.

${ }^{6}$ Kallistratos Ilias, Professor A.T.E.I. Thessaloniki, Greece.

${ }^{7}$ Takidis loannis, Physical Therapist, Greece.

\begin{abstract}
Background: The exponential rise in the population's median age has led to a higher risk of falls among the elderly worldwide. The most frequent complication resulting from falls among older population is bone fracture, especially in the hip, and it increases the likelihood of death in elderly persons. Prevention through improvement of static balance is a necessity for the elderly population. This study examines the effect of a specially designed movement therapy protocol for improving static balance of elderly who have undergone gamma nail surgery to treat a pertrochanteric hip fracture caused by a fall.
\end{abstract}

Methods: The sample consisted of 60 elderly aged 65-74 years, who were randomly divided into two groups: the experimental group $(\mathrm{n}=35)$, which followed an exercise therapy programme covering a total duration of 12 weeks, and the control group $(\mathrm{n}=25)$, which did not follow any programme. Static balance was assessed with the use of a foot analysis platform before and after the intervention so as to determine its effectiveness. A follow-up assessment was performed in six and twelve months after the therapeutic intervention for the purpose of assessing the carry over effect of the results.

Results: The results showed that the experimental group presented a statistically substantial decrease in the fluctuation of the centre of pressure at the end of the therapy programme $(\mathrm{p}<.001)$, as well as 6 months later, on the $\mathrm{x}$ axis $(\mathrm{p}<.001)$ and on the $\mathrm{y}$ axis $(\mathrm{p}<.01)$, and 12 months later $(\mathrm{p}<.001)$, compared to the control group.

Conclusions: The specially designed movement therapy protocol covering a duration of 12 weeks improved the static balance of elderly who have undergone gamma nail surgery to treat a pertrochanteric hip fracture. Keywords: Aged, Accidental Falls, Exercise Therapy, Postural Balance

\section{Introduction}

Older population has significantly increased in the recent years, and the world demographic aging affects not only developing, but also developed countries. By 2050, the world population over 60 years of age is expected to reach 2 billion individuals, who are bound to be more sensitive and vulnerable than normal, regardless of any health conditions [1]. According to the WHO, by 2020 , there will be over 1 billion elderly and by 2050 they will represent $1 / 3$ of the world population [2].

An aged person's quality of life is directly correlated with safeguarding sound functionality and independence [3]. One of the most important problems older population faces is fall- 
ing due to loss of balance. Fall consequences have a decisive impact on human health as well as a country's economic budget [4]. In total, over $30 \%$ of elderly above the age of 65 experience a fall. This rate increases to $40 \%$ among elderly above the age of 80 [5].

The therapeutic community has at its disposal a wide range of fall prevention approaches related to primary prevention and optimisation of quality of life among elderly. However, secondary prevention, i.e. all therapeutic actions focusing on reducing the frequency and severity of complications after the initial fall [6], requires a clinical exercise therapy plan that will aim at improving joint mobility, optimising quality of life and reducing the risk of a new fall.

This is why this paper was undertaken, so as to support hy potheses regarding for the need for secondary prevention after intramedullary nailing surgery (g-nail) in older populations.

\section{Methods}

This study was ethically approved by all the members of the Institutional Review Board that was formed and all the patients participated with a written consent. The sample consisted of 60 elderly citizens aged 65-74 years (old age according to the $\mathrm{WHO}$ ), who had undergone gamma nail surgery to treat a pertrochanteric hip fracture caused by a fall in the last year. Initially, the balance of these people was assessed using the S-Plate portable force plate system. Subsequently, the subjects were randomly divided into two groups by simple randomization, the experimental group $(n=35)$ and the control group $(n=25)$. The experimental group followed an exercise therapy programme covering a total duration of 12 weeks, with each session lasting up to one hour at the most. The control group did not follow any programme. At the end of the programme, the final measurements were carried out on both groups under the same conditions as the initial conditions. Lastly, a follow-up assessment was performed in six and twelve months with the aim of determining the durability of the programme's effect on balance.

The therapeutic plan consisted of a 5-minute warm-up, during which the subjects executed marching while supporting themselves on a chair before each session started. They reached $70 \%$ of their Maximum Heart Rate, they actively stretched their lower extremities for 10 minutes, they performed resistance exercises with the use of middle tension rubber bands for the hip abductor, extensors and flexors, for knee flexors and extensors, as well as ankle dorsiflexor and plantarflexors ( 3 sets of 12-15 repetitions for each muscle group). The performed stretching of the hamstrings, rectus femoris, gluteus maximus, iliopsoas, gastrocnemius, soleus and abdominal-dorsal muscles (10-30 sec/3 times for each muscle group), balance exercises with weight shifts, initially in the seated and gradually in the standing position (first supporting themselves on a chair or a large medicine ball) (for 5 minutes), walking while clapping every 5 steps or looking left and right every 5 steps or lifting the lower extremity higher every 5 steps (for 5 minutes), exercises on a mattress to practice getting out of bed (rolling, side sitting, weight shifting on all fours) (for 10 minutes) and a 5-minute cool-down period with respiratory exercises, rhythmical movement of the upper and lower extremities, and relaxed walking. All the exercises were carried out with the exact order that they are mentioned here.

Computer software SPSS 17.0 (Statistical Package for Social Sciences, Chicago, Illinois, USA) has been used for the statistical processing of the results. Before that, Descriptive Analysis was made. The evaluation of distribution adequacy was accomplished by the use of Kolmogorov-Smirnov test and homogeneity of variances by the use of Levene test.

\section{Results}

In a previous study, balance was defined as the ability to maintain the vertical projection of the body's centre of gravity within the limits set by its base of support, whether it remains stable or moves in space when the body receives external or internal stabilisation stimuli [7].

In order to determine the deviation of the centre of pressure (cop) on the $x$ axis (cop $x$ ) and on the $y$ axis (cop y), repeated measure variance analysis was used (repeated measures ANOVA, $2 \times 4$ ) in relation to two factors, of which only the one was repeated, the dependent variable being the deviation of the centre of pressure (cop $x$ and cop $y$ ), the independent variable being the "group" (experimental group and control group) and the repeated factor being the "measurement" factor (initial - final - 6 months later, 12 months later).

The variance analysis of the two factors showed that there is a statistically significant interaction between the "group" and "measurement" factors, $F(3.58)=108.395 p<0.001$ on the $x$ axis and $F(3.58)=56.213 p<0.001$ on the $y$ axis. A statistically significant main effect occurred in the "measurement" factor, $F(3.58)=105.055 p<0.001$ on the $x$ axis and $F(3.58)=99.264$ $\mathrm{p}<0.001$ on the $y$ axis. Lastly, a statistically significant effect of the "group" factor was also discovered, $F(3.58)=85.412 p<0.001$ on the $x$ axis and $F(3.58)=293.993 p<0.001$ on the $y$ axis.

The Sheffe multiple comparison test was performed in order to determine the groups between which the differences mentioned above are statistically significant. The results showed that the experimental group presented a statistically significant reduction of deviation of cop at the end of the therapy programme $(p<0.001)$, as well as 6 months later, $(p<0.001)$ on the $x$ axis and $(p<0.01)$ on the $y$ axis, and 12 months later, $(p<0.001)$ in relation to the control group. Furthermore, the statistically significant differences remained between the final measurement and that taken 12 months later $(p<0.001)$, as well as between the measurement taken at 6 and 12 months $(p<0.001)$. Statistically significant differences occurred between the experimental group and control group during the final measurement and 6 months later $(p<0.001)$ (Tables 1 and 2 ).

\section{Discussion}

Previous studies have revealed that in elderly individuals, the 
Paris et al. Physical Therapy and Rehabilitation 2017,

Table 1. Deviation of the center of pressure on the $\mathrm{x}$ axis (mean \pm SD).

\begin{tabular}{lllll}
\hline & \multicolumn{2}{l}{$\begin{array}{l}\text { Experimental } \\
\text { Group }(\mathbf{n}=35)\end{array}$} & \multicolumn{2}{l}{$\begin{array}{l}\text { Control } \\
\text { Group }(\mathbf{n}=25)\end{array}$} \\
\hline & mean & SD & mean & SD \\
\hline Initial measurement & 6.80 & 0.12 & 6.84 & 0.34 \\
Final measurement & 6.23 & 0.22 & 6.86 & 0.17 \\
6 months post-intervention & 6.50 & 0.31 & 6.89 & 0.26 \\
12 months post-intervention & 6.88 & 0.21 & 6.74 & 0.35 \\
\hline
\end{tabular}

Dependent Variables

Table 2. Deviation of center of pressure on axis y (mean $\pm S D)$.

\begin{tabular}{|c|c|c|c|c|}
\hline & \multicolumn{2}{|c|}{$\begin{array}{l}\text { Experimental } \\
\text { Group }(n=35)\end{array}$} & \multicolumn{2}{|c|}{$\begin{array}{l}\text { Control } \\
\text { Group }(n=25)\end{array}$} \\
\hline & mean & SD & mean & SD \\
\hline Initial measurement & 6.08 & 0.05 & 6.04 & 0.34 \\
\hline Final measurement & 5.23 & 0.51 & 6.06 & 0.17 \\
\hline 6 months post-intervention & 5.32 & 0.23 & 6.04 & 0.26 \\
\hline 12 months post-intervention & 5.28 & 0.15 & 5.92 & 0.35 \\
\hline
\end{tabular}

deterioration of balance is compensated by the increase in oscillation $[8,9]$. The results of this study have shown that the exercise therapy programme for the elderly leads to a statistically significant reduction in the oscillation of the centre of pressure, both on the frontal ( $\operatorname{cop} x$ ) and on the anteroposterior plane (cop y). Furthermore, it appears that the effects of the therapy programme present high levels of durability six and twelve months after the therapy programme has ended.

The organic changes observed in the functional systems of the body as a result of ageing lead to a differentiation in balance between young adults and old adults [10]. The latter is achieved through the following three strategies (including gait), of which two are the most prevalent: 1) the ankle strategy and 2) the hip strategy [11]. The ankle strategy is used for balance in cases of destabilising stimuli of low intensity and frequency, while the hip strategy is used for counterbalancing stimuli of high intensity and frequency. The way the above strategies are used according to ageing.

A previous study examined the ways in which young and old people react and adjust their posture during static balance activities of increasing difficulty by recording the centre of pressure, as this study does [12]. The results of this study show that older people rely more on the hip strategy, thus significantly increasing the risk of falling, which is not the case with young people. This study seems to complement the above findings by clearly indicating that a programme focusing on improving strength, balance and mobility of the hip improves - by extension-the changes in the centre of pressure of seniors who have undergone gamma nail surgery to treat a pertrochanteric hip fracture caused by a fall. The reduced base of support of participants in this study appears to increase oscillation due to the surgery, however they respond very positively to a combined therapeutic approach, possibly forcing them to use a mixed strategy (combination of hip and ankle strategy) in order to maintain their balance $[13,14]$.

\section{Conclusions}

In conclusion, the results of this study indicate clearly that the combined movement therapy programme has a positive effect on balance and, by extension, on the secondary prevention of falls in elderly who have undergone gamma nail surgery to treat a pertrochanteric hip fracture caused by a fall in the last year. It should be underlined that the improvements achieved through the application of the therapy protocol appear to have a high carry over effect both 6 and 12 months after completion.

\section{Competing interests}

The authors declare that they have no competing interests.

Authors' contributions

\begin{tabular}{|l|c|c|c|c|c|c|c|}
\hline Authors' contributions & PTI & AT & MN & MS & KS & KI & TI \\
\hline Research concept and design & $\checkmark$ & $\checkmark$ & $\checkmark$ & -- & $\checkmark$ & $\checkmark$ & -- \\
\hline Collection and/or assembly of data & $\checkmark$ & $\checkmark$ & $\checkmark$ & -- & $\checkmark$ & -- & $\checkmark$ \\
\hline Data analysis and interpretation & $\checkmark$ & $\checkmark$ & -- & $\checkmark$ & $\checkmark$ & -- & -- \\
\hline Writing the article & $\checkmark$ & -- & -- & $\checkmark$ & -- & $\checkmark$ & $\checkmark$ \\
\hline Critical revision of the article & $\checkmark$ & -- & $\checkmark$ & $\checkmark$ & -- & $\checkmark$ & $\checkmark$ \\
\hline Final approval of article & $\checkmark$ & $\checkmark$ & $\checkmark$ & -- & -- & -- & $\checkmark$ \\
\hline Statistical analysis & $\checkmark$ & -- & -- & $\checkmark$ & $\checkmark$ & $\checkmark$ & -- \\
\hline
\end{tabular}

\section{Acknowledgements}

This work was supported by the staff of Kilkis General Hospital. We would like to thank them for the voluntary assistance. In addition, we appreciate the effort of all the patients that participated and provided a healthy collaboration.

\section{Publication history}

Editor: Mohammad H. Hadadzadeh, Wheeling Jesuit University, USA.

Received: 05-Jul-2017 Final Revised: 23-Sep-2017

Accepted: 06-Nov-2017 Published: 19-Nov-2017 


\section{References}

1. Bozzetti F. Nutritional issues in the care of the elderly patient. Crit Rev Oncol Hematol. 2003; 48:113-21. | Article | PubMed

2. World Health Organization National Institute on Aging National Institutes of Health U.S. Department of Health and Human Services October 2011.

3. Oliver D, Daly F, Martin FC and McMurdo ME. Risk factors and risk assessment tools for falls in hospital in-patients: a systematic review. Age Ageing. 2004; 33:122-30. | Article | PubMed

4. Sattin RW, Lambert Huber DA, DeVito CA, Rodriguez JG, Ros A, Bacchelli $S$, Stevens JA and Waxweiler RJ. The incidence of fall injury events among the elderly in a defined population. Am J Epidemiol. 1990; 131:1028-37. | PubMed

5. Tinetti ME. Performance-oriented assessment of mobility problems in elderly patients. J Am Geriatr Soc. 1986; 34:119-26. | Article | PubMed

6. Unsworth J. Falls in older people: the role of assessment in prevention and care. Br J Community Nurs. 2003; 8:256-62. | Article | PubMed

7. Horak FB, Shupert $\mathrm{CL}$ and Mirka A. Components of postural dyscontrol in the elderly: a review. Neurobiol Aging. 1989; 10:727-38. | PubMed

8. Era P, Heikkinen E, Gause-Nilsson I and Schroll M. Postural balance in elderly people: changes over a five-year follow-up and its predictive value for survival. Aging Clin Exp Res. 2002; 14:37-46. | PubMed

9. Maki BE, Edmondstone MA and Mcllroy WE. Age-related differences in laterally directed compensatory stepping behavior. J Gerontol A Biol Sci Med Sci. 2000; 55:M270-7. | PubMed

10. Streepey JW and Angulo-Kinzler RM. The role of task difficulty in the control of dynamic balance in children and adults. Hum Mov Sci. 2002; 21:423-38. | Article | PubMed

11. Horak FB, Nashner LM and Diener HC. Postural strategies associated with somatosensory and vestibular loss. Exp Brain Res. 1990; 82:167-77. I PubMed

12. Amiridis IG, Hatzitaki V and Arabatzi F. Age-induced modifications of static postural control in humans. Neurosci Lett. 2003; 350:137-40. I Article I PubMed

13. Hatzitaki V, Amiridis IG and Arabatzi F. Aging effects on postural responses to self-imposed balance perturbations. Gait Posture. 2005; 22:250-7. | Article | PubMed

14. lakovidis Paris P.T, Msc K. E, Kofotolis Nikolaos G.A, and Apostolou Thomas M.D. The Efficacy of Kinesiotherapy in Secondary Fall Prevention among seniors for Optimisation of their Health Quality. International Journal of Health Sciences. 2016; 4:41-45.

\section{Citation:}

Paris IT, Nikolaos M, Stavros K, Savvas M, Thomas A, Ilias $\mathrm{K}$ and Ioannis $\mathrm{T}$. The effect of physiotherapeutic intervention on the static balance of elderly for secondary prevention of falls. Phys Ther Rehabil. 2017; 4:15. http://dx.doi.org/10.7243/2055-2386-4-15 